\title{
Strain effect on electrical conductance of p-type nanocrystalline silicon carbide thin film
}

\author{
Hoang-Phuong Phan, ${ }^{* a}$ Dzung Viet Dao, ${ }^{a, b}$ Li Wang, ${ }^{a}$ Toan Dinh,${ }^{a}$ Nam-Trung Nguyen, ${ }^{a}$ \\ Afzaal Qamar, ${ }^{a}$ Philip Tanner, ${ }^{a}$ Sima Dimitrijev, ${ }^{a, b}$ and Yong Zhu ${ }^{a, b}$
}

Received * * 2014

DOI: $10.1039 / b 000000 x$

This paper presents for the first time the effect of strain on the electrical conductance of p-type nanocrystalline $\mathrm{SiC}$ grown on a $\mathrm{Si}$ substrate. The gauge factor of the p-type nanocrystalline $\mathrm{SiC}$ was found to be 14.5 which is one order of magnitude larger than that in most metals. This result indicates that mechanical strain has a significant influence on electrical conduction of p-type nanocrystalline $\mathrm{SiC}$, which is promising for mechanical sensing applications in harsh environments.

Silicon carbide (SiC) with its large energy band gap (2.3-3.4 $\mathrm{eV}$ ) and excellent mechanical properties is a promising material for electrical devices operating in harsh environments ${ }^{1-3}$. Recently, researchers have paid significant attention to the characterization of the strain effect on $\mathrm{SiC}$ for sensing applications at high temperatures. Many groups have reported the piezoresistive effect in various poly types of single crystalline $\mathrm{SiC}$ such as $3 \mathrm{C}-\mathrm{SiC}, 4 \mathrm{H}-\mathrm{SiC}$, and $6 \mathrm{H}-\mathrm{SiC}$. Large gauge factors of approximately 30 were reported in both p-type and n-type single crystalline $\mathrm{SiC}$, indicating that $\mathrm{SiC}$ is a good candidate for mechanical sensing applications ${ }^{4-11}$. Among more than 200 poly types of crystalline $\mathrm{SiC}$, cubic crystalline silicon carbide (3C-SiC) is preferable for Micro Electro Mechanical Systems (MEMS) transducers. The main advantage of $3 \mathrm{C}-\mathrm{SiC}$ is the capability of growth on a silicon $(\mathrm{Si})$ substrate, which reduces the cost of wafers and is more compatible with the conventional MEMS process ${ }^{3,9-12}$. Nevertheless, $\mathrm{Si}$ is not a suitable material for hostile environment applications due to the current leakage from the epitaxy $\mathrm{SiC}$ layer to the Si substrate at high temperatures. Therefore, wafer bonding techniques are required to transfer single $3 \mathrm{C}-\mathrm{SiC}$ to other electrical insulating substrates, which can withstand high temperatures (e.g silicon dioxide and silicon nitride) ${ }^{13}$. This process indeed increases the cost of wafers as well as complicates the fabrication of $\mathrm{SiC}$ devices.

Different from single crystalline $\mathrm{SiC}$, nanocrystalline $\mathrm{SiC}$ (nc-SiC), with its grain size in sub-micron scale, can be grown on various substrates (e.g. silicon, silicon dioxide, silicon nitride) and therefore, it is a good candidate for MEMS trans-

\footnotetext{
* Email of corresponding author: hoangphuong.phan@griffithuni.edu.au

a Queensland Micro-Nanotechnology Centre, Griffith University, Queensland, Australia.

${ }^{b}$ School of Engineering, Griffith University, Queensland, Australia.
}

ducers ${ }^{14-16}$. Nanocrystalline $\mathrm{SiC}$ can be grown by chemical vapor deposition (CVD) at $1100^{\circ} \mathrm{C}^{15}$, sputtering amorphous $\mathrm{SiC}$ at approximately $750^{\circ} \mathrm{C}$ followed by thermal annealing ${ }^{17}$, and in situ crystallization of amorphous $\mathrm{SiC}$ at $600^{\circ} \mathrm{C}^{18}$. Recent studies also demonstrated the possibility of synthesis nc$\mathrm{SiC}$ at room temperature using the supersonic molecular beam epitaxy ${ }^{19}$. The capability of growth at low temperatures on various substrates not only simplifies the fabrication, but also allows MEMS surface processing in nc-SiC. Electrical properties of p-type nc-SiC have been intensively investigated in the last decades for applications involving the next generation of solar cells ${ }^{20,21}$. Nanocrystalline SiC films also have been utilized as an electrode material for electrochemical and biochemical applications ${ }^{16,22}$. However, to the best of our knowledge, no study has reported the piezoresistive effect of p-type nc-SiC for MEMS mechanical sensing applications.

In this paper, we report for the first time the piezoresistive effect of p-type nc-SiC. The insight gained in this study is not only important for the development of nc-SiC based mechanical sensors, but also valuable for understanding the impact of mechanical strain on nc-SiC based devices such as solar cells which are affected by various types of stress during the fabrication process and in applications.

The SiC films were deposited on n-type (100) Si wafers using the low pressure chemical vapor deposition (LPCVD) method at a low temperature of $600^{\circ} \mathrm{C}^{18}$. Prior to deposition, standard RCA cleaning was carried out to clean the surface of the $\mathrm{Si}$ wafers. Methylsilane $\left(\mathrm{H}_{3} \mathrm{SiCH}_{3}\right)$ was used as a single precursor in a hot wall LPCVD reactor. Trimethylaluminium (TMA, $\left.\mathrm{Al}\left(\mathrm{CH}_{3}\right)_{3}\right)$ with a flow rate of $0.15 \mathrm{SCCM}$ was introduced as a means of doping p-type SiC. Aluminum ( $\mathrm{Al}$ ) was utilized as the acceptor atom in this in situ doping process due to its shallow ionization energy of approximately $200 \mathrm{meV}$. Xray Photoelectron Spectroscopy (XPS) analysis showed that the concentration of $\mathrm{Al}$ was 4.1 at. \%, while the $\mathrm{Si} / \mathrm{C}$ ratio was 0.68 .

The microstructures of deposited SiC films were characterized by transmission electron microscopy (TEM) and x-ray diffractometry (XRD). The thickness of the nc-SiC films was $240 \mathrm{~nm}$ and their surface roughness was approximately $6 \mathrm{~nm}$. Figure 1 shows a typical SAED pattern and High Resolution TEM (HRTEM) image. Multi-rings with spots are observed in the SAED pattern, whereas the arrayed diffraction spots are 
(a)

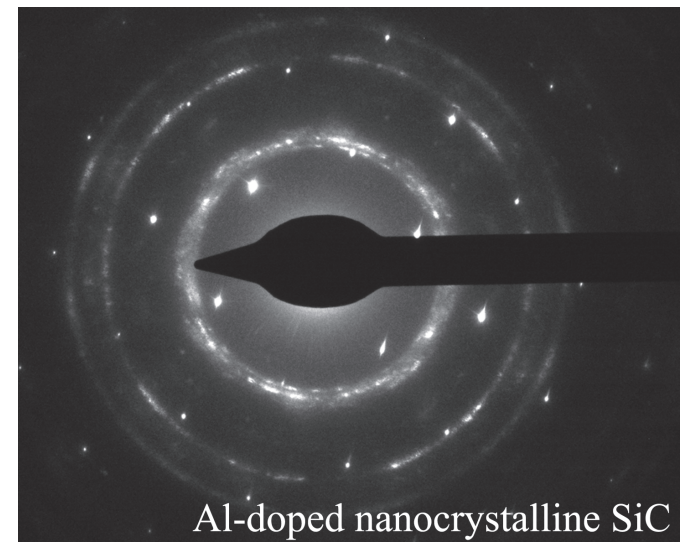

(b)
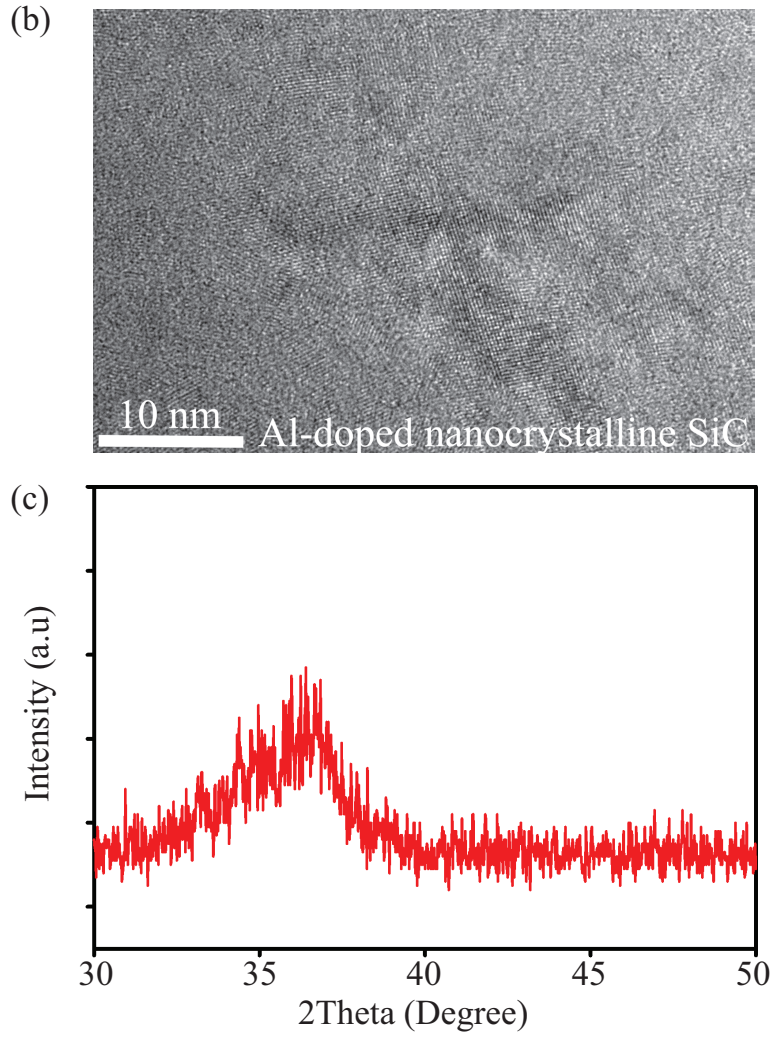

Fig. 1 Optical characterization of nc-SiC: a) SAED pattern-the spotty rings indicate that the film contains randomly oriented nc-SiC grains; the arrayed diffraction spots are from underlying $\mathrm{Si}$ substrate; b) Cross-sectional HRTEM image-the presence of randomly oriented lattice grains and fringes also confirm that the film is made of nc-SiC; c) X-Ray diffraction pattern-the broad diffraction peak indicates that the the $\mathrm{SiC}$ grains are relatively small (average size around $13.4 \mathrm{~nm}$ ) and exhibit some degree of misorientation. The residual stress in the film can also cause the broadening of the diffraction peak. (Fig 1. (a) and (b): Reproduced with permission from ${ }^{18}$. Copyright [2009], AIP Publishing LLC.)

from the Si substrate beneath (Fig. 1 (a)). These spotty rings and lattice fringes (as shown in Fig. 1(b)), indicate that the deposited film is composed of $\mathrm{SiC}$ nano grains with different ori- entations ${ }^{18}$. The broad diffraction peak at around $36.2^{\circ}$ from $\theta-2 \theta$ XRD scan indicates that the $\mathrm{SiC}(111)$ grains are relatively small and exhibit some degree of misorientation (Fig. 1(c)) . This result also suggests that the film might include 3C-SiC(111), 4H-SiC(004), 6H-SiC(102), and other SiC poly types, which can contribute to a diffraction peak at this $2 \theta$ position. Additionally, the residual stress in the film can also cause the broadening of the diffraction peak. According to Debye-Scherrer equation ${ }^{23}$, the average $\mathrm{SiC}$ grain size was found to be around $13.4 \mathrm{~nm}$. The capacitance-voltage $(\mathrm{C}-\mathrm{V})$ measurements demonstrated that the $\mathrm{SiC}$ is p-type conductive with a doping concentration of $2 \times 10^{18} \mathrm{~cm}^{-3}$.

To investigate the piezoresistive effect in nc-SiC, we patterned $\mathrm{SiC}$ resistors on $\mathrm{Si}$ substrate using a conventional photolithography process ${ }^{9}$. The size of the $\mathrm{SiC}$ resistors was $100 \mu \mathrm{m} \times 300 \mu \mathrm{m} \times 240 \mathrm{~nm}$. Aluminum was used as the electrodes of the resistors. The current-voltage (I-V) curve of the $\mathrm{SiC}$ resistors shows a linear relationship between the applied voltage and measured current. Accordingly, the resistivity of the nc-SiC was found to be approximately $72 \Omega \mathrm{cm}^{-1}$ which is 3 orders of magnitude larger than that of a single crystalline $3 \mathrm{C}-\mathrm{SiC}$ film at the same carrier concentration ${ }^{10,11}$. This indicates that the grain boundary of $\mathrm{nc}-\mathrm{SiC}$ significantly increases the electrical resistance of nc-SiC films. As the p-type nc-SiC was grown on n-type $\mathrm{Si}$, it is important to investigate the contribution of Si substrate to the measurement of the piezoresistive effect in nc-SiC. The pn junction between p-type nc$\mathrm{SiC}$ and n-type $\mathrm{Si}$ creates a depletion layer at $\mathrm{SiC} / \mathrm{Si}$ interface, preventing the leakage-current from $\mathrm{SiC}$ to $\mathrm{Si}$ layer ${ }^{9,24}$. The leakage-current through $\mathrm{SiC} / \mathrm{Si}$ heterojunction was measured to be $2 \%$ of the current flowing in $\mathrm{SiC}$ resistors. This indicates that, the substrate contributed $2 \%$ of the measured conductance of nc-SiC, which needs to be considered when calculating the gauge factor of nc-SiC. To lower the influence of substrate, we aligned the n-type Si substrate in [110] orientation, which has a smaller piezoresistive effect than [100] orientation $^{25-27}$.

The $\mathrm{SiC}$ wafers with $\mathrm{SiC}$ resistors were then diced into strips with a dimension of $60 \mathrm{~mm} \times 9 \mathrm{~mm} \times 0.6 \mathrm{~mm}$. The bending beam method was then employed to induce strain on nc-SiC film (Fig. 2). As the thickness of nc-SiC film is much smaller than that of the $\mathrm{Si}$ substrate, the strain of $\mathrm{SiC}$ can be approximately equal to that of the top surface of the Si beam, using the following equation:

$$
\varepsilon=\frac{M t}{E I}
$$

where $M$ is the bending moment; $t$ is the distance from the nc$\mathrm{SiC}$ layer to the neutral axis of the Si beam; $E$ is the Young's modulus of $\mathrm{Si}$; and $I$ is the inertia moment of the Si beam. Accordingly, with an applied load varying from 0 to $2 \mathrm{~N}$, the induced strain ranged from 0 to $800 \mathrm{ppm}$.

The resistance change of the $\mathrm{SiC}$ resistor during the bending experiment was measured by the 4 points measurement using the Agilent ${ }^{\mathrm{TM}} 34410 \mathrm{~A}$ Multimeter. To reduce the influence of 
(a)

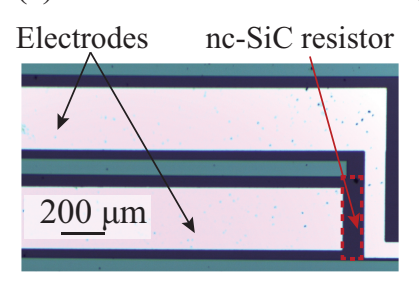

(b)

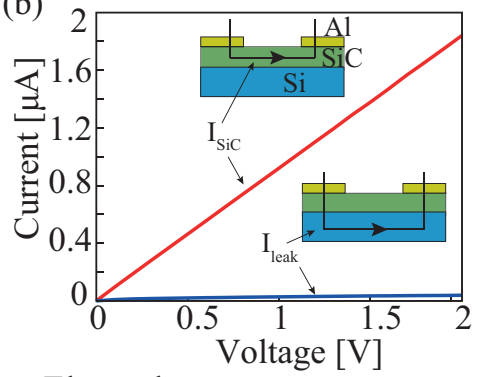

(c)

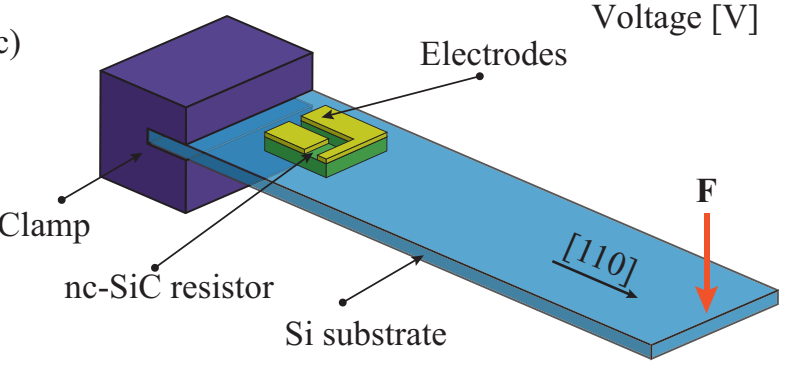

Fig. 2 (a) Photograph of a nc-SiC resistor. (b) The I-V curve of nc-SiC resistor. (c) Schematic sketch of the bending experiment.

the Joule's heating effect, the resistance of nc-SiC was measured at a low current of $500 \mathrm{nA}$ and a consumption power of below $0.25 \mu \mathrm{W}$. Figure 3 shows the relationship between the applied strain and the relative resistance change of p-type $\mathrm{nc}-\mathrm{SiC}$, indicating that the resistance of $\mathrm{nc}-\mathrm{SiC}$ increased proportionally with increasing strain. The gauge factor of $\mathrm{nc}-\mathrm{SiC}$ is calculated as:

$$
G F=\frac{\Delta R_{\mathrm{SiC}} / R_{\mathrm{SiC}}}{\varepsilon}=-\frac{\Delta \sigma_{\mathrm{SiC}} / \sigma_{\mathrm{SiC}}}{\varepsilon}
$$

where $R_{\mathrm{SiC}}, \sigma_{\mathrm{SiC}}$ are the resistance and conductance of nc$\mathrm{SiC}$, respectively. Since there is a current leakage from nc$\mathrm{SiC}$ layer to the Si substrate (approximately $2 \%$ of the current flowing through the $\mathrm{SiC}$ resistor), the influence of the piezoresistive effect of Si substrate and $\mathrm{SiC} / \mathrm{Si}$ heterojunction is necessarily taken into account. Let $\sigma_{\text {sub }}$ be the conductance of the substrate. The measured conductance (or resistance) is the sum of $\mathrm{SiC}$ conductance and substrate conductance:

$$
\sigma=\sigma_{\mathrm{SiC}}+\sigma_{\mathrm{sub}}
$$

Therefore, the relative conductance change is:

$$
\begin{aligned}
\frac{\Delta \sigma}{\sigma}=\frac{\Delta \sigma_{\mathrm{SiC}}+\Delta \sigma_{\mathrm{sub}}}{\sigma} & =\frac{\sigma_{\mathrm{SiC}}}{\sigma} \frac{\Delta \sigma_{\mathrm{SiC}}}{\sigma_{\mathrm{SiC}}}+\frac{\sigma_{\mathrm{sub}}}{\sigma} \frac{\Delta \sigma_{\mathrm{sub}}}{\sigma_{\text {sub }}} \\
& =\frac{98}{100} \frac{\Delta \sigma_{\mathrm{SiC}}}{\sigma_{\mathrm{SiC}}}+\frac{2}{100} \frac{\Delta \sigma_{\mathrm{sub}}}{\sigma_{\text {sub }}}
\end{aligned}
$$

We fabricated a reference resistor to investigate the relative conductance change of the substrate during bending experiment. At an applied strain of $800 \mathrm{ppm}$ on the $\mathrm{SiC}$ resistor, the relative conductance change of the substrate was found to be $0.1 \%$. As a result, from Eq. 2 and Eq. 4, the substrate only

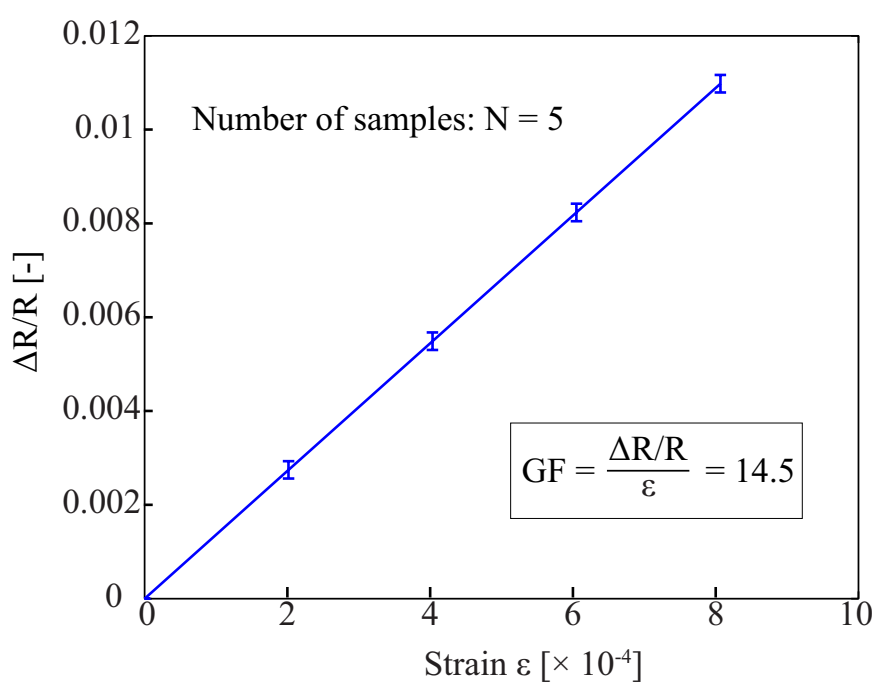

Fig. 3 The relationship between the relative resistance change of nc-SiC and applied strains.

contributed $0.2 \%$ to the measured gauge factor, which is negligible. Thus, the gauge factor of p-type nc-SiC was found to be 14.5, which is much larger than that in most metals ${ }^{27-31}$.

We explain the piezoresistive effect in p-type nc-SiC using the model of current transport in several polycrystalline and nanocrystalline materials ${ }^{32-34}$, in which p-type nc-SiC contains several crystalline grains, connected to each other by grain boundaries. For the sake of simplification, we describe the structure of $\mathrm{nc}-\mathrm{SiC}$ in one dimension (1-D), as shown in Fig. 4. Based on this model, the total resistance of nc-SiC is the sum of grains' resistance $\left(R_{\mathrm{C}}\right)$ and resistance of boundary $\left(R_{\mathrm{b}}\right)$ :

$$
R_{\mathrm{nc}-\mathrm{SiC}}=R_{\mathrm{c}}+R_{\mathrm{b}}
$$

When a strain is applied, both resistance of grains and boundaries change, causing the change of the total resistance.

Crystalline grains aligned in arbitrary orientations

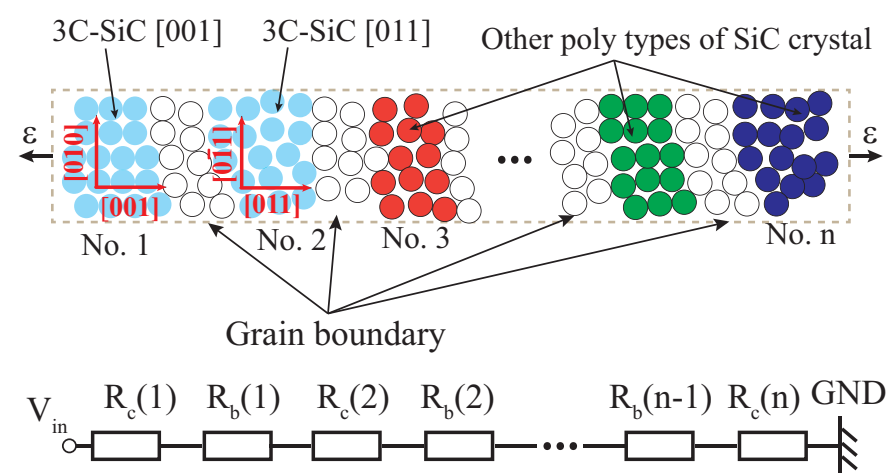

Fig. 4 An one dimensional (1-D) model of the crystalline structure of nc-SiC which contains a large number of crystalline $\mathrm{SiC}$ grains and grain-boundary. 
Firstly, we considered the piezoresistive effect in crystalline $\mathrm{SiC}$ grains. Based on the deformation potential model, the piezoresistive effect in p-type semiconductors is due to the splitting of heavy holes and light holes in the valence band under applied strains ${ }^{27,35}$. This band splitting leads to the redistribution of heavy holes and light holes in these two bands, which follows the rule that holes will fill lower energy levels. The re-population of holes results in the change of electrical resistivity of the crystalline $\mathrm{SiC}$.

Next, we took the effect of strain to the resistance of grain boundaries into account. The resistance of boundaries can be explained based on the tunneling current between neighbor grains. At grain boundaries, atoms have twisted/strained and/or dangling bonds. These defects result in the formation of trapping states which immobilize charge carriers, creating a potential barrier $\left(V_{\mathrm{b}}\right)^{32,33}$. Consequentially, the carriers can move through these potential barriers via the tunneling current which is described in the following equation ${ }^{36,37}$ :

$$
J \propto p_{\mathrm{hh}} J_{\mathrm{hh}}+p_{\mathrm{lh}} J_{\mathrm{lh}}
$$

where $p_{\mathrm{hh}}$ and $p_{\mathrm{lh}}$ are the concentration of heavy hole and light hole; and $J_{\mathrm{hh}}$ and $J_{\mathrm{lh}}$ are tunneling current components of heavy hole and light hole which depend on the potential barrier $\left(\propto e^{q V_{\mathrm{b}} / k T}\right.$, where $q$ is electron charge; $V_{\mathrm{b}}$ is the potential barrier; $k$ is the Boltzmann constant; and $T$ is the absolute temperature). Under strain, heavy holes and light holes concentrations change. As a consequence, the tunneling current varies with applied strain. Thus, the piezoresistive effect of nc-SiC grain boundaries in fact is established from the piezoresistive effect of each nc-SiC single crystal.

It is well-known that the piezoresistive effect in single crystalline $\mathrm{SiC}$ depends on crystallography orientations. As such, in our previous work on the characterization of the piezoresistive effect in p-type $3 \mathrm{C}$-SiC, we reported a large longitudinal gauge factor of 30.3 in [110] orientation, and a smaller longitudinal gauge factor of 5.0 in [100] orientation of single crystalline $3 \mathrm{C}-\mathrm{SiC}^{10}$. Therefore, if the applied strain does not align with the orientation corresponding to the most significant piezoresistive effect of a single crystal, the gauge factor is smaller. In other words, the random arrangement of crystalline grains in $\mathrm{nc}-\mathrm{SiC}$ may have contributed to the diminution of the gauge factor of $\mathrm{nc}-\mathrm{SiC}$ in comparison to that of single crystalline $\mathrm{SiC}^{33}$. Additionally, the grain boundary scattering is expected to play an important role in decreasing the gauge factor of polycrystalline and nanocrystalline materials ${ }^{38}$.

In conclusion, we characterized the piezoresistive effect of p-type nanocrystalline SiC grown by the LPCVD process at a low temperature of $600^{\circ} \mathrm{C}$. At a carrier concentration of $2 \times$ $10^{18} \mathrm{~cm}^{-3}$, the gauge factor of p-type nc-SiC was found to be 14.5 , which is larger than metals, but smaller than single crystalline SiC. This result was explained due to the change of the resistivity of crystal grains and boundaries as well as the randomly aligned orientations of SiC crystals. Due to the capability of growing on various substrate (e.g. $\mathrm{Si}, \mathrm{SiO}_{2}$ ) at a lower temperature than single crystalline $\mathrm{SiC}$, the significant piezoresistive effect in nc-SiC makes it a good candidate for MEMS mechanical sensors used in harsh environments and bio applications.

This work was performed in part at the Queensland node of the Australian National Fabrication Facility, a company established under the National Collaborative Research Infrastructure Strategy to provide nano and micro-fabrication facilities for Australia's researchers. This work has been partially supported by the Griffith University's New Researcher Grants.

\section{References}

1 P. M. Sarro, Sens. Actuators. A, 2000, 82, 210.

2 X. Zhang, Y. Chen, W. Liu, W. Xue, J. Li and Z. Xie, J. Mater. Chem. C, 2013, 1, 6479-6486.

3 M. Mehregany, C. A. Zorman, N. Rajan and C. H. Wu, Proc. IEEE, 1998, 86, 1594.

4 J. Bi, G. Wei, L. Wang, F. Gao, J. Zheng, B. Tang and W. Yang, J. Mater. Chem. C, 2013, 1, 4514.

5 F. Gao, J. Zheng, M. Wang, G. Wei and W. Yang, Chem. Commun., 2011, 47, 11993.

6 R. Shao, K. Zheng, Y. Zhang, Y. Li, Z. Zhang and X. Han, Appl. Phys. Lett., 2012, 101, 233109.

7 T. Akiyama, D. Briand and N. F. Rooij, J. Micromech. Microeng., 2012, 22, 085034.

8 R. S. Okojie, A. A. Ned, A. D. Kurtz and W. N. Carr, IEEE Trans. Electron Devices, 1998, 45, 785.

9 H. P. Phan, P. Tanner, D. V. Dao, N. T. Nguyen, L. Wang, Y. Zhu and S. Dimitrijev, IEEE Electron Device Lett., 2014, 35, 399.

10 H. P. Phan, D. V. Dao, P. Tanner, N. T. Nguyen, L. Wang, Y. Zhu and S. Dimitrijev, Appl. Phys. Lett., 2014, 104, 111905.

11 H. P. Phan, D. V. Dao, P. Tanner, N. T. Nguyen, J. S. Han, S. Dimitrijev, G. Walker, L. Wang and Y. Zhu, J. Matter. Chem. C, 2014, 2, 7176-7179.

12 J. S. Shor, D. Goldstein and A. D. Kurtz, IEEE Trans. Electron Devices, 1993, 40, 1093.

13 C. H. Wu, C. A. Zorman and M. Mehregany, IEEE Sensors J., 2006, 6, 316.

14 Y. Komura, A. Tabata, T. Narita, M. Kanaya, A. Kondo and T. Mizutani, Jpn. J. Appl. Phys., 2007, 46, 45-50.

15 B. Somogyi, V. Zolyomi and A. Gali, Nanoscale, 2012, 4, 7720-7726.

16 M. Eickhoff, M. Moller, G. Kroetz and M. Stutzmann, J. Appl. Phys., 2004, 96, 2872-2879.

17 N. Naderi and M. R. Hashim, Matter. Lett., 2013, 97, 9092.

18 L. Wang, S. Dimitrijev, P. Tanner and J. Zou, Appl. Phys. Lett., 2009, 94, 181909.

19 R. Verucchi, L. Aversa, M. V. Nardi, S. Taioli, S. Beccara, 
D. Alfe, L. Nasi, F. Rossi, G. Salviati and S. Iannotta, J. Am. Chem. Soc., 2012, 134, 17400-17403.

20 S. Y. Myong and K. S. Lim, Appl. Phys. Lett., 2005, 86, 033506.

21 S. Miyajima, M. Sawamura, A. Yamada and M. Konagai, J. Non-Cryst. Solids, 2008, 354, 2350.

22 N. Yang, H. Zhuang, R. Hoffmann, W. Smirnov, J. Hees, X. Jiang and C. E. Nebel, Anal. Chem., 2011, 83, 58275830.

23 H. Tateyama, H. Noma, Y. Adachi and M. Komatsu, Chem. Mater., 1997, 9, 766-772.

24 A. Qamar, P. Tanner, D. V. Dao, H. P. Phan and T. Dinh, IEEE Electron Device Lett., 2014, 35, 1293-1295.

25 M.-D. Nguyen, H.-P. Phan, K. Matsumoto and I. Shimoyama, Proc. $26^{\text {th }}$ IEEE Int. Conf. Micro Electro Mechanical Systems (MEMS 2013), 2013, 617-620.

26 D. V. Dao, T. Toriyama, J. Wells and S. Sugiyama, Proc. $15^{\text {th }}$ IEEE Int. Conf. Micro Electro Mechanical Systems (MEMS 2002), 2002, 20-24.

27 A. A. Barlian, W. T. Park, J. R. M. Jr., A. J. Rastegar and B. L. Pruitt, Proc. IEEE, 2009, 97, 513.

28 J. Engel, J. Chen and C. Liu, J. Micromech. Microeng., 2003, 13, 359-366.

29 W. K. Schomburg, Z. Rummler, P. Shao, K. Wulff and
L. Xie, J. Micromech. Microeng., 2004, 14, 1101-1108.

30 I. H. Kazi, P. M. Wild, T. N. Moore and M. Sayer, Thin Solid Films, 2006, 515, 2602-2606.

31 D. Macnamara, D. Thiel, D. James and P. Lisner, Proc. SPIE 6035, Microelectronics: Design, Technology and Packaging II, 2006, 603524.

32 V. Mosser, J. Suski, J. Goss and E. Obermeier, Sensors Actuators A: Physical, 1991, 28, 113-132.

33 P. Alpuim, M. Andrade, V. Sencadas, M. Ribeiro, S. Filonovich and S. Lanceros-Mendez, Thin Solid Films, 2007, 515, 7658-7661.

34 S. D. Janssens, S. Drijkoningen and K. Haenen, Appl. Phys. Lett., 2014, 105, 101601.

35 K. Nakamura, D. V. Dao, Y. Isono, T. Toriyama and S. Sugiyama, Advanced Inorganic Chemistry, In-Tech, Vienna, Austria, 2010.

36 X. Liu, C. Shi and R. Chuai, Polycrystalline silicon piezoresistive nano thin film technology, InTech, Croatia, 2009.

37 C. Chuai, B. Liu, X. Liu, X. Sun, X. Shi and L. Wang, J. Semicond., 2010, 31, 032002.

38 L. Fang, W. L. Wang, P. D. Ding, K. J. . Liao and J. Wang, J. Appl. Phys., 1999, 86, 5185-5193. 